\title{
Time-resolved diffuse optical tomography system based on adaptive structured light illumination and compressive sensing detection
}

\author{
Andrea Farina $^{\mathrm{a}}$, Marta Betcke ${ }^{\mathrm{b}}$, Andrea Bassi ${ }^{\mathrm{a}, \mathrm{c}}$, Gianluca Valentini $^{\mathrm{a}, \mathrm{c}}$, Simon Arridge ${ }^{\mathrm{b}}$, and \\ Cosimo D'Andrea ${ }^{\mathrm{c}, \mathrm{d}}$ \\ ${ }^{a}$ Consiglio Nazionale delle Ricerche, Istituto di Fotonica e Nanotecnologie, Piazza Leonardo da \\ Vinci 32, 20133, Milano, Italy \\ ${ }^{\mathrm{b}}$ Centre for Medical Image Computing, University College London, Malet Place, London \\ WC1E 6BT, UK \\ 'Politecnico di Milano, Dipartimento di Fisica, Piazza L. da Vinci 32, 20133 Milano, Italy \\ ${ }^{\mathrm{d}}$ Center for Nano Science and Technology@PoliMi, Istituto Italiano di Tecnologia, 20133 \\ Milan, Italy
}

\begin{abstract}
Diffuse Optical Tomography (DOT)is a powerful tool for the reconstruction of optical properties inside a diffusive medium, such as biological tissues. In particular, in the last years, techniques based on structured light illumination and compressive sensing detection have been developed. In this work a time-resolved system based on structured light illumination and compressive detection has been developed and used for DOT. Moreover, a data-driven algorithm for optimal pattern generation based on the Singular-Value Decomposition has been implemented and validated.
\end{abstract}

Keywords: Diffuse Optical Tomography, structured light, adaptive algorithm, time-resolved, compressive sensing

\section{INTRODUCTION}

Diffuse Optical Tomography (DOT) is a biomedical imaging technique capable to reconstruct a three-dimensional map of the optical properties (absorption and scattering coefficients) from measurements taken the boundary of the tissue sample. Heterogeneities can be ascribed, as an example, to alterations in oxygen saturation (oxymetry) and to the presence of a lesion (breast mammography). ${ }^{1}$ The classical approach to DOT is based on a dense coverage of source/detector pairs placed on the surface. ${ }^{2}$ This dense coverage is usually achieved using point-like sources and single-element detector, making the system quite complex and expensive. Moreover, time-resolved detection has been demonstrated to increase the accuracy of the optical properties measurement and, if used in a gated approach, also to increase spatial resolution when "early photons", i.e. photon having run a short path in the tissue, are distinguished from "late photon", i.e. photon having run a long path. ${ }^{3}$ Thus, the high-density requirement and the time-resolved capability are very difficult to co-exist due to the complexity of the timing electronics on the picoseconds scale. A possibility to overcome this limitation is the use of structured light illumination and compressive detection. Together with the fact that images collected after photon propagation inside a scattering medium have a limited bandwidth, it is possible to envisage compression approaches devoted to data-set reduction without information-loss using time-resolved patterned light illumination and single-pixel camera detection. ${ }^{4,5}$

In the recent years there is an increasing attention to adaptive algorithms in which measurement results drive new acquisitions strategies in real-time. ${ }^{6-9}$ This is important for reducing both acquisition and computation times. In the field of DOT this can be achieved using a wide-field approach both in the input and output sides.

Further author information: (Send correspondence to Andrea Farina)

Andrea Farina: E-mail: andrea.farina@polimi.it 


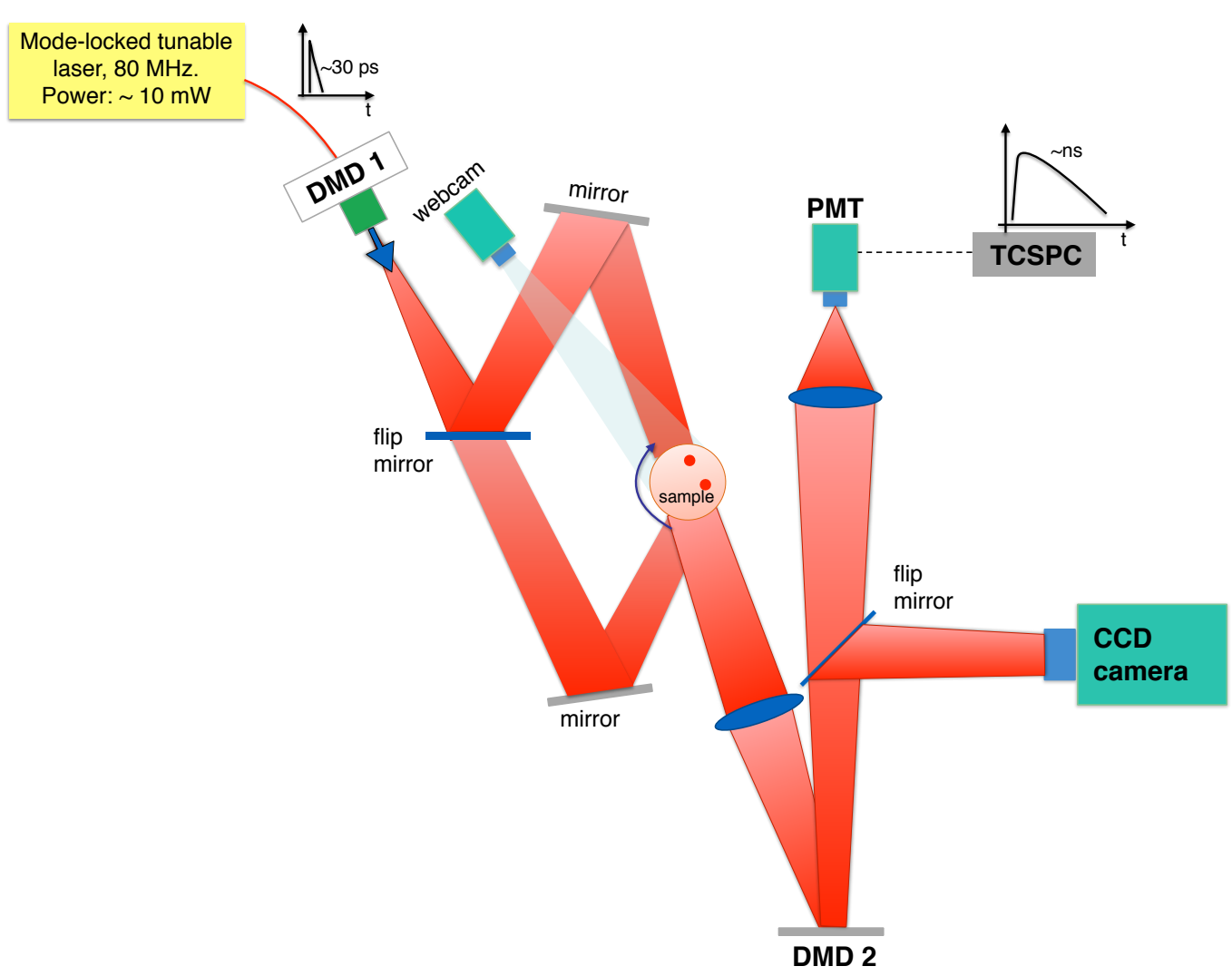

Figure 1. Experimental setup. In the figure the direction of light is depicted in red. After the first DMD (DMD 1), patterned light is projected on the sample either in reflection or in transmission geometry by means of a flip mirror. On the detection side, after the second DMD (DMD 2), the light can either be focused on a Photomultiplier Tube (PMT) for time-resolved detection or on a CCD camera, by means of a second flip mirror.

In the present work a system for time-resolved DOT is described and validated on a cylindrical phantom containing absorbing heterogeneities using Walsh-Hadamard patterns. Afterwards, an adaptive algorithm for optimal pattern search based on the Singular-Value Decomposition (SVD) has been devised and validated on a slab phantom.

\section{MATERIAL AND METHODS}

The setup is depicted in Fig.1. A supercontinuum fiber laser source (SC-450, Fianium) provides mode-locking pulses $(40 \mathrm{MHz})$ in the spectral range $450-1750 \mathrm{~nm}$. The collimated output first passes through a short-pass filter

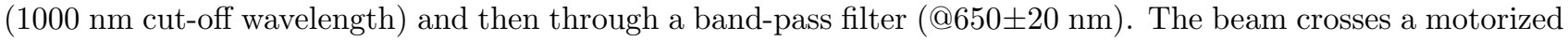
circular neutral-density filter for attenuation (4 OD) and then is coupled to a graded-index fiber $(\varnothing=100 \mu \mathrm{m})$ by means of an objective lens (40x). The fiber output is then delivered to an on-axis projection system based on a Total-Internal Reflection (TIR) prism coupled to a Digital Micromirror Device (DMD) (Discovery kit 1100, Vialux) for patterned illumination. After the projection system, an achromatic lens ( $\mathrm{f}=75 \mathrm{~mm}$ ) projects patterns on the input plane of the sample. This is achieved by means of a flip mirror and a second mirror for the capability to work both in reflectance and transmittance modalities. The sample is mounted on a rotational motorized stage for multiple-view acquisition. The output plane of the sample is then imaged on a second DMD device (Discovery kit 4100, Vialux) for implementation of the single-pixel camera. The DMD plane is then optically conjugated to either a cooled CCD (Versarray 512, Princeton Instruments) or to a step-index optical fiber tip $(\varnothing=1 \mathrm{~mm})$ through a long-working distance objective lens $(10 \mathrm{x})$. The two paths can be switched by means of a flip mirror. The step-index fiber is finally connected to an imaging system based on two achromatic lenses for coupling the light to a photomultiplier tube (PMT) (HPM-100-50, Becker \& Hickl). This PMT is connected 
to a Time-Correlated Single-Photon Counting (TCSPC) board (SPC-630, Becker \& Hickl) for time-resolved acquisition. The synchronism signal is directly provided by an electrical output on the laser source.

The overall Instrumental-Response Function (IRF) of the system, taking into account all broadening due to propagation in fibers and optical materials is about 200 ps FWHM. To measure the IRF, a peace of white paper has been put in the middle of the input and output plane. The two cameras are used for calibration purposes in the following way: on a DMD some control pixels are sequentially switched on at known positions. For each point an image is acquired with the camera and the corresponding fired pixel is detected. Afterwards, a geometric transformation is computed between the DMD space and the camera space, which in turns enables the possibility to correctly project any pattern on the target plane. This procedure is applied on both the DMDs. Illumination and detection field-of-view are about $20 \times 20 \mathrm{~mm}^{2}$.

The overall measurement is controlled by a home-made software managing both the DMDs, the rotational stage, the camera acquisition and the TCSPC board. The software is capable to work in a slave modality for adaptive measurements. In this case MATLAB runs as the server triggering the measurements.

The tomographic capabilities of the system have been first tested on a cylindrical tissue mimicking phantom

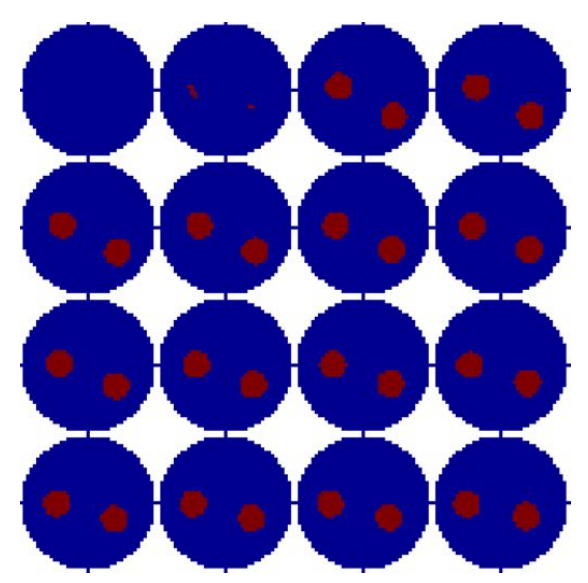

(a)

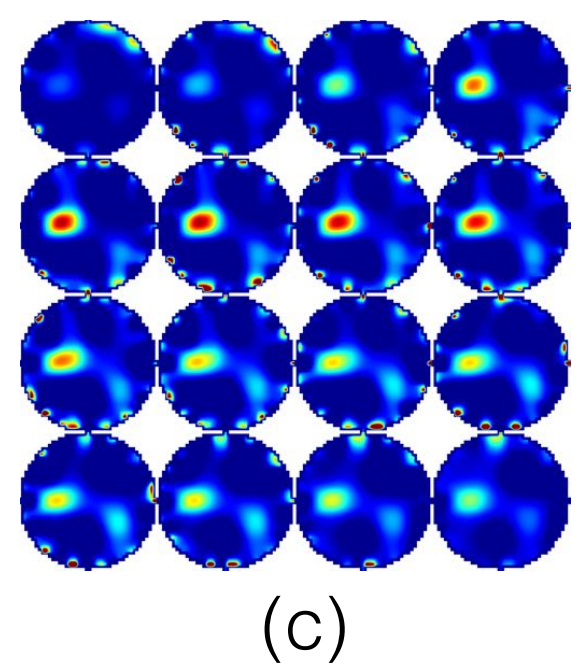

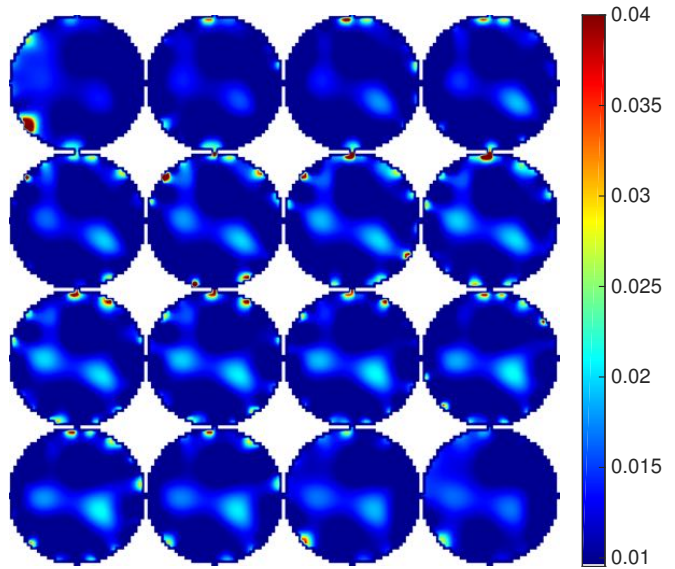

(b)

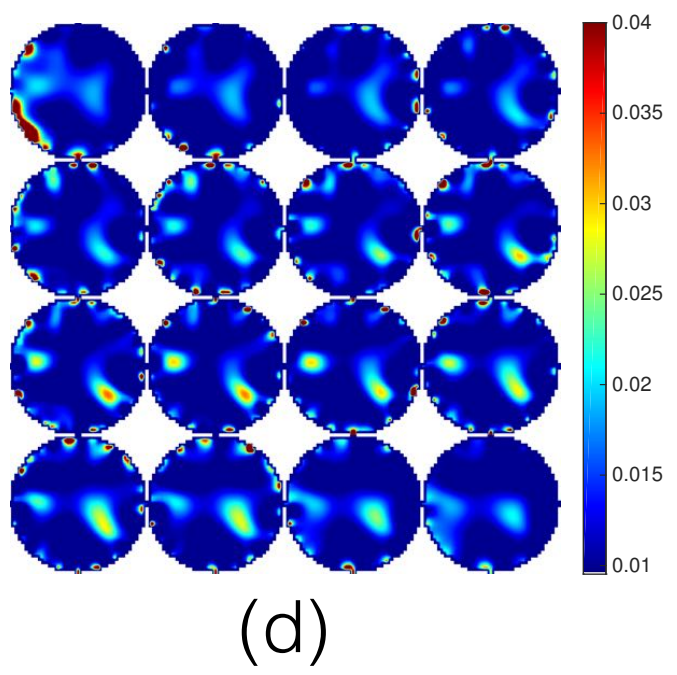

Figure 2. Reconstructions using multiple-view acquisition and Walsh-Hadamard patterns for three absorption cases Slices along the cylinder axis at $1 \mathrm{~mm}$ step are shown. (a) reference, (b) $\mu_{a}=0.05 / 0.05 \mathrm{~mm}^{-1}$ for both the inclusions, (c) $0.05 / 0.1 \mathrm{~mm}^{-1}$ and (d) $0.05 / 0.03 \mathrm{~mm}^{-1}$. The reduced scattering coefficient is the same of the background $\left(1 \mathrm{~mm}^{-1}\right)$. 
$\left(\varnothing=20 \mathrm{~mm}\right.$, height $50 \mathrm{~mm}$ ) made of epoxy resin, $\mathrm{TiO}_{2}$ (as a scatterer), and a toner (as an absorber). Background optical properties $\left(\mu_{a}=0.01 \mathrm{~mm}^{-1}\right.$ and $\mu_{s}^{\prime}=1 \mathrm{~mm}^{-1}$ ) were measured by means of a time-resolved spectroscopy system. ${ }^{10}$ Heterogeneities can be added by filling two holes $(\varnothing=1.6 \mathrm{~mm})$ drilled into the sample with a calibrated solution of intralipid (as a scatterer) and black ink (as an absorber). ${ }^{11}$ Different combinations of absorption perturbations have been tested. 8x8 Walsh-Hadamard patterns on the detection side and planar illumination, for each view ( 8 views at $45^{\circ}$ ), have been used. ${ }^{12}$ A nonlinear Gauss-Newton reconstruction algorithm has been applied with a Total Variation regularizer under the framework of TOAST++, a Finite-Element based software for time-resolved light diffusion forward and inverse problems. ${ }^{13,14}$ Time-resolved data integrated on an early gate (width $400 \mathrm{ps}$ ) before the peak has been used. ${ }^{3}$ More details on the data analysis can be found in reference. ${ }^{5}$ Results are shown in Fig. 2 for three absorption combinations.

After this first validation we decided to better understand the importance of input/output patterns related to the heterogeneity position in the sample and explore the possibility of an optimal pattern generation algorithm based on the Singular-Value Decomposition (SVD). To achieve this we used a slab phantom (15 x $\left.64 \times 64 \mathrm{~mm}^{3}\right)$ with the same background optical properties $\left(\mu_{a}=0.01 \mathrm{~mm}^{-1}, \mu_{s}^{\prime}=1 \mathrm{~mm}^{-1}\right)$ with two holes drilled inside the phantom at slightly different depths with respect to the two squared surfaces. A cylindrical black rod has been placed in the holes alternatively on the input and output sides. To find optimal patterns both for the input and the output side, Singular-Value Decomposition (SVD) has been applied. In particular, a contrast matrix $C$ has been constructed with a sequence of differential CCD images (with and without perturbation) acquired when a point illumination on the input side raster scans the illumination area. It is worth noting that the input pattern set can be any basis, such as Walsh-Hadamard, Wavelet, Fourier. By performing SVD of the matrix $C$ it is possible to retrieve a set of input $\left(\mathbf{v}_{i}\right)$ and output $\left(\mathbf{u}_{i}\right)$ vectors (i.e. patterns) ordered by the singular-values $\lambda_{i}$ that, in turns, give the respective weight of the two i-th input/output vectors. In particular the decomposition is as follow:

$$
C=U \Lambda V^{T}
$$

in which the columns of $\mathrm{U}$ are $\mathbf{u}_{i}$, the columns of $\mathrm{V}$ are $\mathbf{v}_{i}$ and $\Lambda$ is a diagonal matrix containing the singularvalues $\lambda_{i}$ ordered by their magnitude. Due to magnitude-ordered characteristic of the singular values $\lambda_{i}$, it is possible to truncate the spectrum to a certain order $k$ and reconstruct an approximated version $C_{k}$ given by:

$$
C_{k}=U \Lambda_{k} V^{T}
$$

where $\Lambda_{k}$ is the reduced singular-values diagonal matrix. It is worth noting that no positivity constrain in the SVD is imposed, thus for every pattern two positive patterns were implemented on the DMDs after proper area normalization. A truncation to the fifth order is enough for good reconstruction.

Optimal patterns were then projected to the sample and time-resolved single-pixel camera acquisition was enabled. It is interesting to show (see Fig. 3) that the first pattern for every sample well represents the shape and position of the perturbation. When the inclusion is on the output side, output patterns shapes around the inclusion whereas the input pattern is almost unstructured. The contrary happens when the inclusion is on the input side.

\section{CONCLUSIONS}

In this work, we have shown and characterized a time-resolved DOT system based on structured illumination and compress sensing detection. In particular, after a first validation of the tomographic capabilities of the system using a multiple-view approach on a cylindric phantom, we have shown the importance of both the input/output patterns related to the position of the inclusions using an SVD-based optimal pattern algorithm. Results show that optimal patterns present a structure quite similar to the perturbation inside. In particular, the structure is mostly present on the pattern set related to the side in which the perturbation is more close to the external surface. This is a simple scheme for an adaptive algorithm in which patterns are online calculated during measurement and can set the basis for future developments on more complicated geometry phantoms.

\section{ACKNOWLEDGMENTS}

We thankfully acknowledge funding from Laserlab-Europe EU-H2020 654148 and the support of NVIDIA with the donation of the Tesla K40 GPU used for this research. 


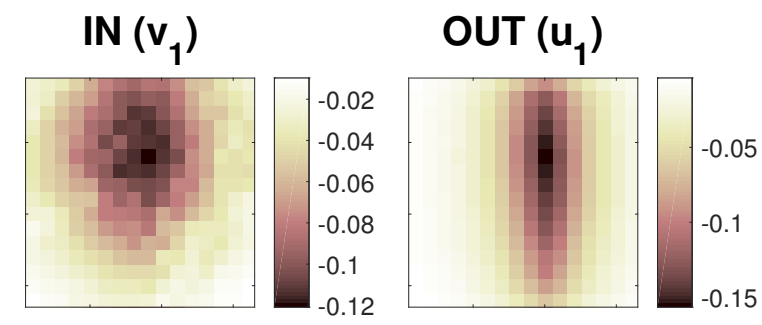

(a)

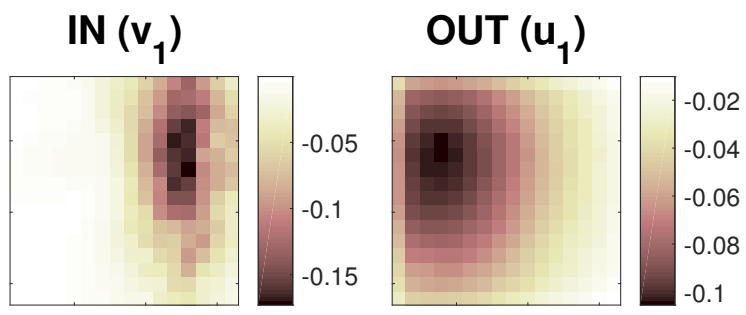

(b)

Figure 3. First optimal patterns obtained by SVD decomposition. (a) heterogeneity on the output side, (b) heterogeneity on the input side. It is possible to see how the shape of the first SVD pattern well represents the shape of the inclusion. These patterns are derived directly by the SVD, thus their L2-norm is one. For each of them, two versions of positive patterns have been generated for implementation in the DMDs devices.

\section{REFERENCES}

[1] Durduran, T., Choe, R., Baker, W. B., and Yodh, A. G., "Diffuse optics for tissue monitoring and tomography," Reports on Progress in Physics 73, 1-43 (2010).

[2] Eggebrecht, A. T., Ferradal, S. L., Robichaux-Viehoever, A., Hassanpour, M. S., Dehghani, H., Snyder, A. Z., Hershey, T., and Culver, J. P., "Mapping distributed brain function and networks with diffuse optical tomography," Nature Photonics 8, 448-454 (5 2014).

[3] Bassi, A., D'Andrea, C., Valentini, G., Cubeddu, R., and Arridge, S., "Temporal propagation of spatial information in turbid media.," Optics letters 33, 2836-2838 (2008).

[4] Bassi, A., D'Andrea, C., Valentini, G., Cubeddu, R., and Arridge, S., "Detection of inhomogeneities in diffusive media using spatially modulated light.," Optics letters 34(14), 2156-2158 (2009).

[5] Farina, A., Betcke, M., di Sieno, L., Bassi, A., Ducros, N., Pifferi, A., Valentini, G., Arridge, S., and DAndrea, C., "Multiple-view diffuse optical tomography system based on time-domain compressive measurements," Optics Letters 42(14), 2822 (2017).

[6] Soldevila, F., Salvador-Balaguer, E., Clemente, P., Tajahuerce, E., and Lancis, J., "High-resolution adaptive imaging with a single photodiode," Scientific reports 5, 14300 (2015).

[7] Tian, L., Liu, Z., Yeh, L.-H., Chen, M., Zhong, J., and Waller, L., "Computational illumination for highspeed in vitro Fourier ptychographic microscopy," Optica 2(10) (2015).

[8] Rousset, F., Ducros, N., Farina, A., Valentini, G., D'Andrea, C., and Peyrin, F., "Adaptive Basis Scan by Wavelet Prediction for Single-pixel Imaging," IEEE transaction on computational imaging 3(1), 36-46 (2016).

[9] Rousset, F., Ducros, N., Peyrin, F., Valentini, G., DAndrea, C., and Farina, A., "Time-resolved multispectral imaging based on an adaptive single-pixel camera," Optics Express 26(8), 10550 (2018).

[10] Konugolu Venkata Sekar, S., Dalla Mora, A., Bargigia, I., Martinenghi, E., Lindner, C., Farzam, P., Pagliazzi, M., Durduran, T., Taroni, P., Pifferi, A., and Farina, A., "Broadband (600-1350 nm) Time-Resolved Diffuse Optical Spectrometer for Clinical Use," IEEE Journal on Selected Topics in Quantum Electronics 22(3), 406-414 (2016).

[11] Spinelli, L., Botwicz, M., Zolek, N., Kacprzak, M., Milej, D., Sawosz, P., Liebert, A., Weigel, U., Durduran, T., Foschum, F., Kienle, A., Baribeau, F., Leclair, S., Bouchard, J.-P., Noiseux, I., Gallant, P., Mermut, O., Farina, A., Pifferi, A., Torricelli, A., Cubeddu, R., Ho, H.-C., Mazurenka, M., Wabnitz, H., Klauenberg, K., Bodnar, O., Elster, C., Bénazech-Lavoué, M., Bérubé-Lauzière, Y., Lesage, F., Khoptyar, D., Subash, a. a., Andersson-Engels, S., Di Ninni, P., Martelli, F., and Zaccanti, G., "Determination of reference values for optical properties of liquid phantoms based on Intralipid and India ink," Biomedical Optics Express 5, 2037-2053 (6 2014).

[12] Huynh, N., Zhang, E., Betcke, M., Arridge, S., Beard, P., and Cox, B., "Single-pixel optical camera for video rate ultrasonic imaging," Optica 3(1), 26 (2016). 
[13] Schweiger, M. and Arridge, S., "The Toast ++ software suite for forward and inverse modeling in optical tomography," Journal of biomedical optics 19(4), 1-15 (2014).

[14] Schweiger, M., Arridge, S. R., and Nissilä, I., "Gauss-Newton method for image reconstruction in diffuse optical tomography," Physics in Medicine and Biology 50(10), 2365-2386 (2005). 